POS $\quad$ PROCEEDINGS

\title{
Constraints on charged Higgs bosons in the CMSSM and NUHM models
}

\section{Oscar Stål*}

High-Energy Physics, Dept. of Physics and Astronomy, Uppsala University

P.O. Box 535, SE-751 21 Uppsala, Sweden

E-mail: oscar.stalephysics.uu.se

In the context of the MSSM scenarios CMSSM (constrained MSSM) and NUHM (Non-Universal Higgs Masses) we discuss experimental constraints on charged Higgs bosons. The parameter regions in $\left(m_{H^{+}}, \tan \beta\right)$ favored by data from flavour physics experiments and LEP mass limits are confronted with the projected sensitivities of ATLAS and CMS. We find that the sensitivity of the LHC experiments to discover the charged Higgs is highest in the same region of parameter space where the strongest constraints are obtained by indirect experiments.

Prospects for Charged Higgs Discovery at Colliders

September 16-19 2008

Uppsala, Sweden

${ }^{*}$ Speaker. 


\section{Introduction}

The charged Higgs boson appearing in two-Higgs doublet models (such as in the MSSM) has a special status as an additional mediator of charged current interactions. It can therefore contribute already at tree-level to several rare decays. The charged Higgs boson contribution to different observables can therefore be used in order to put constraints on the MSSM Higgs sector. This has been the topic of our recent work [1] reported on here. ${ }^{1}$

\section{Charged Higgs bosons in the MSSM}

In the Minimal Supersymmetric Standard Model (MSSM), the Higgs sector takes on a specific form. In addition to the weak $\mathrm{SU}(2)_{\mathrm{L}}$ Higgs doublet $\Phi_{1}$ of the Standard Model (SM), another doublet $\Phi_{2}$ is introduced. Supersymmetry requires the superpotential to be analytic in $\Phi_{1}, \Phi_{2}$, which forces the two doublets to have opposite hypercharges. In this way a discrete $Z_{2}$ symmetry differentiating $\Phi_{1}$ from $\Phi_{2}$ is introduced naturally as a requirement of supersymmetry. This results in a Yukawa sector of "Type II" - meaning only one of the doublets couples to the up-type fermions, whereas the other doublet couples exclusively to the down-type fermions. Since the remaining parameters in the Higgs potential are fixed by gauge couplings, the MSSM Higgs sector is completely described at tree-level by two parameters. Following electroweak (EW) symmetry breaking, the masses of the physical Higgs states $\left(h, H, A, H^{ \pm}\right)$are therefore related. A convenient choice for the two parameters is the mass of the $C P$-odd Higgs $m_{A}$, or of the charged Higgs boson $m_{H^{+}}$, together with the ratio of vacuum expectation values $\tan \beta \equiv\left\langle\Phi_{2}\right\rangle /\left\langle\Phi_{1}\right\rangle$. Choosing either of the two masses as input is completely equivalent, since at tree-level $m_{H^{+}}^{2}=m_{A}^{2}+m_{W}^{2}$.

\section{MSSM GUT models}

Since the full ( $R$-parity conserving) MSSM has 124 free parameters, the theory is not suitable for phenomenology without a scheme for reducing this number. To begin with, one therefore imposes so-called Minimal Flavor Violation (MFV), which amounts to saying there should be no flavour structure in the MSSM beyond that of the CKM framework. This is a reasonable assumption, especially given the strict constraints on that exist on flavour-changing neutral currents, but the lack of a theoretical motivation for MFV can still be seen as unsatisfactory.

The two observations that i) supersymmetry allows for gauge coupling unification, and ii) the breaking of supersymmetry must be mediated at a scale lying much above the EW scale, has directed interest towards scenarios with unifying boundary conditions for the fundamental parameters at a very high scale. One such scenario is the constrained MSSM (CMSSM), which is a four-parameter description of the MSSM. At the unification scale, the universal parameters in this scenario are the scalar mass $m_{0}$, the gaugino mass $m_{1 / 2}$, the trilinear coupling $A_{0}$, the sign of the Higgsino mass parameter $\mu$, and finally $\tan \beta$ as defined above. Note in particular that the Higgs mass parameter $m_{A}$ is not free parameter in this scenario. In the NUHM models, the universality

\footnotetext{
${ }^{1}$ Due to space limitations, we cannot cite all the interesting work published on constraints in the MSSM. We apologize for this fact, and refer to [1] for an extensive list of references.
} 


\begin{tabular}{cccl}
\hline Parameter & $\min$ & $\max$ & note \\
\hline$m_{0}$ & 50 & 2000 & \\
$m_{1 / 2}$ & 50 & 2000 & \\
$A_{0}$ & -2000 & 2000 & \\
$\tan \beta$ & 1 & 60 & \\
$\mu$ & -2000 & 2000 & CMSSM: only sign \pm \\
$m_{A}$ & 5 & 600 & NUHM only \\
\hline
\end{tabular}

Table 1: Ranges for the input parameters used in the CMSSM and NUHM scans. The parameters $m_{0}, m_{1 / 2}$, $A_{0}$, and $\tan \beta$ are specified at the unification scale, whereas $\mu$ and $m_{A}$ are given at the EW scale. All values, except $\tan \beta$, are given in $\mathrm{GeV}$.

\begin{tabular}{lrrr}
\hline Constraint & Experimental value & SM value & Allowed range \\
\hline $\mathrm{BR}\left(B \rightarrow X_{s} \gamma\right)$ & $(3.52 \pm 0.23 \pm 0.09) \times 10^{-4}$ & $(3.15 \pm 0.22) \times 10^{-4}$ & {$[2.15,4.89] \times 10^{-4}$} \\
$\Delta_{0}\left(B \rightarrow X_{s} \gamma\right)$ & $(3.1 \pm 2.3) \times 10^{-2}$ & $(8.4 \pm 2.1) \times 10^{-2}$ & {$[-1.7,8.9] \times 10^{-2}$} \\
$\mathrm{BR}\left(B_{u} \rightarrow \tau v_{\tau}\right)$ & $(1.41 \pm 0.43) \times 10^{-4}$ & $(1.10 \pm 0.29) \times 10^{-4}$ & {$[0.58,2.23] \times 10^{-4}$} \\
$\xi\left(B \rightarrow D \tau v_{\tau}\right)$ & $(41.6 \pm 11.7 \pm 5.2) \times 10^{-2}$ & $(29 \pm 3) \times 10^{-2}$ & {$[15.1,68.1] \times 10^{-2}$} \\
$\mathrm{BR}\left(B_{s} \rightarrow \mu^{+} \mu^{-}\right)$ & $<5.8 \times 10^{-8}$ & $(3.2 \pm 0.5) \times 10^{-9}$ & $<6.6 \times 10^{-8}$ \\
$R_{l 23}\left(K \rightarrow \mu v_{\mu}\right)$ & $1.004 \pm 0.007$ & 1 & {$[0.990,1.018]$} \\
\hline
\end{tabular}

Table 2: Values for flavour physics observables used to obtain constraints on charged Higgs bosons. Allowed ranges, including the MSSM contributions, are given at $95 \% \mathrm{CL}$. Some of the observables are described briefly in the text. For references, and details on how the precise numbers are obtained, see [1,3].

of the Higgs doublet mass parameters is relaxed and separated from $m_{0}$, introducing two additional parameters with dimension of mass. We take these to be the values of $\mu$ and $m_{A}$ at the EW scale.

The full set of MSSM parameters (masses, couplings) at the EW scale is obtained from the fundamental parameters at the unification scale through renormalization group evolution. We use the code SOFTSUSY [2] for this part. Selecting reasonable values for the input parameters - the precise ranges can be found in table 1 - we perform scans over the parameter spaces in the CMSSM and NUHM scenarios to study the charged Higgs boson in more detail.

\section{Constraints}

Present experimental data on several observables from flavour physics can be used to constrain the possible contributions from a charged Higgs boson. In table 2 we list the different observables we consider, their current experimental values, the theoretical value obtained in the SM, and finally the 95\% CL allowed range in an MSSM calculation. For a detailed description of the various contributions to each observable, and how these numbers were obtained, we refer the reader to [1]. To clarify the notation, let us just mention that $\Delta_{0}$ is the degree of isospin asymmetry in $B \rightarrow X_{s} \gamma$ transitions, the quantity $\xi$ is defined as $\xi_{D l v_{l}} \equiv \mathrm{BR}\left(B \rightarrow D \tau v_{\tau}\right) / \mathrm{BR}\left(B \rightarrow D e v_{e}\right)$, and that the definition of $R_{l 23}$ in $K \rightarrow \mu v$ decays can be found in [1]. For the theoretical evaluation of these observables, both in the SM and in the MSSM, we use Super I so [3].

To determine the values of $m_{H^{+}}$and $\tan \beta$ which are in agreement with the constraints, the observables are evaluated for the points from the parameter scan. Adding the experimental and 

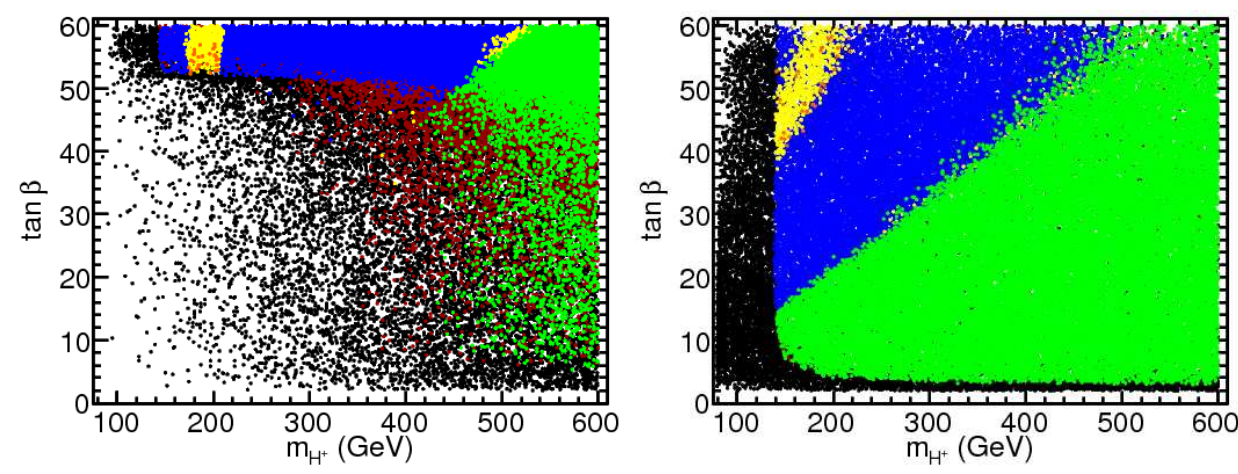

- Allowed

- Direct

- $b \rightarrow s \gamma$

- $\mathrm{B}_{\mathrm{u}} \rightarrow \tau v$

$\mathrm{B}_{\mathrm{s}} \rightarrow \mu^{+} \mu$

- $\mathrm{B} \rightarrow \mathrm{D} \tau \mathrm{v}$

$=\mathrm{K} \rightarrow \mu \mathrm{v}$

Figure 1: Model points from the CMSSM (left) and NUHM (right) confronted with current experimental data. The multi-dimensional parameter space is projected on the $\left(m_{H^{+}}, \tan \beta\right)$ plane. Model points allowed by the constraints are plotted in the foreground (green). Exclusion by different constraints is illustrated by different colors (see legend). The constraints are applied in the order they appear in the legend.

theoretical errors in quadrature, the allowed range at 95\% CL is determined separately for each observable. Treating the statistics in a simplistic manner, we denote a model point from the scan excluded if it generates a value for one or more of the observables outside the allowed ranges. A point for which all values fall in the $95 \%$ allowed regions is termed allowed. When interpreting the constraints from the flavour data, a general caveat applies about (sometimes large) parametric uncertainties, e.g. from $V_{u b}$, which are not included in the quoted numbers.

The main reference [1] contains a large number of plots illustrating the exclusion separately for each constraint. Here we show only the combined result on $\left(m_{H^{+}}, \tan \beta\right)$, given in figure 1 . These plots are obtained by applying the constraints listed in table 2 in sequence. In addition to these constraints, the limits from LEP on Higgs and sparticle masses have been applied. For the mass $m_{h}$ of the lightest Higgs boson, the limit $m_{h}>111 \mathrm{GeV}$ is used. Points excluded by the the LEP constraints are shown in black.

From figure 1, we note first that the region of low $m_{H^{+}}$in the CMSSM is only accessible at high $\tan \beta$ as a result of the RGE running. In the NUHM the full space is covered with points since $m_{H^{+}}$is essentially a free parameter. We further note that in both scenarios the region of low $m_{H^{+}}$ and high $\tan \beta$, where the $H^{+}$couples strongly to the $b$ quark, appears excluded by the combination of flavor physics constraints. A particularly large region is seen to be excluded by the results from $B_{u} \rightarrow \tau v_{\tau}$ transitions, which is understandable since this decay is mediated by the charged Higgs already at tree-level. For such observables, the sparticles play only a minor role through higher order corrections. Contrasting this striking feature is the result on $b \rightarrow s \gamma$ transitions, which are known to be important for constraining charged Higgs bosons in the pure two-Higgs doublet model. We see that, at least in the NUHM scenario, the $H^{+}$contribution can easily be balanced out by the contribution of charginos-neutralinos, resulting in the poor exclusion power of $b \rightarrow s \gamma$ in this case.

To summarize, we find in the CMSSM that the combined constraints exclude charged Higgs masses below $400 \mathrm{GeV}$, while for the NUHM scenario this limit is lower, corresponding to $m_{H^{+}} \gtrsim$ $135 \mathrm{GeV}$ for $\tan \beta \sim 10$. This result reflects the greater freedom offered by the two additional free parameters in this scenario. 

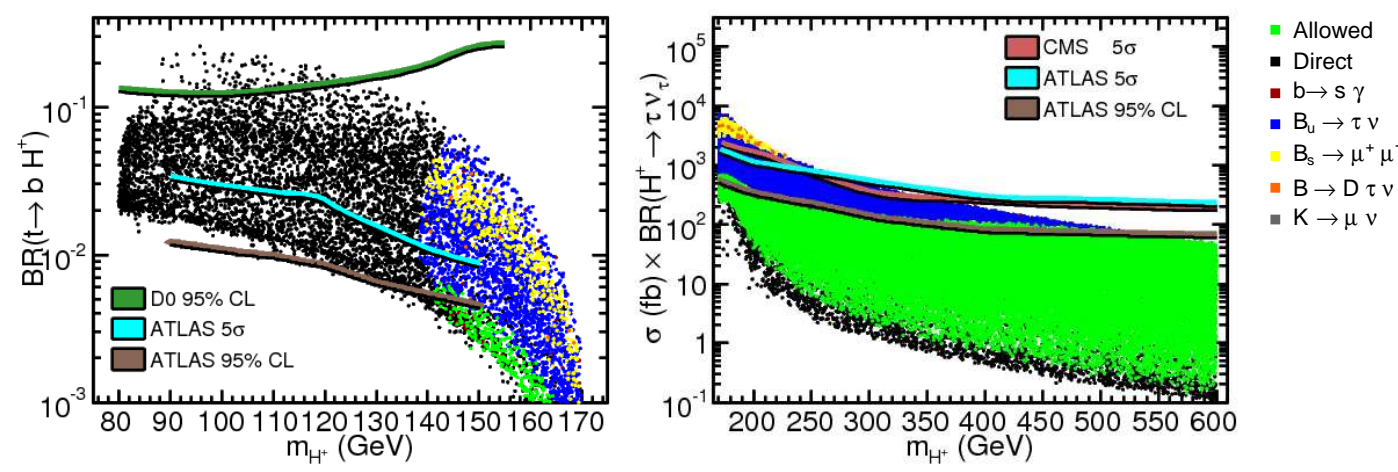

Figure 2: Charged Higgs production cross section and branching ratios for points from the NUHM scan, compared to the reach of ATLAS and CMS. The experimental contours correspond to the projected sensitivities to discover, or exclude, the charged Higgs boson with a given cross section and BR for $30 \mathrm{fb}^{-1}$ of integrated luminosity.

\section{LHC prospects}

To assess the prospects of the LHC experiments to discover the charged Higgs boson present in these constrained MSSM scenarios, we compare the experimental reach reported by ATLAS [4], and CMS [5], for $30 \mathrm{fb}^{-1}$ to the charged Higgs production cross section we obtain for the points in the scan. In this part we focus solely on the NUHM scenario. We divide the comparison into two cases, treating the light $\left(m_{H^{+}}<m_{t}\right)$ and the heavy $\left(m_{H^{+}}>m_{t}\right)$ charged Higgs separately. The light charged Higgs is primarily produced in the decay $t \rightarrow b H^{+}$of on-shell top quarks, whereas the heavy $H^{+}$is produced in association with a single top quark. For both cases, the only decay mode we consider is $H^{+} \rightarrow \tau^{+} v_{\tau}$, since this channel has proven most viable experimentally. This mode is also the channel analyzed for the experimental results we compare to.

The results are shown in figure 2, where it can be seen the that the current best limit on $\mathrm{BR}\left(t \rightarrow b H^{+}\right)$by D0 [6] is just about to start probing the interesting region at low masses. It can also be seen that the ATLAS experiment will have good sensitivity to the NUHM models in most of this mass region. For the heavy case, the situation is slightly worse, and we see that the region with a cross section high enough for discovery is largely excluded by the indirect constraints.

These model-independent discovery contours in figure 2 are translated into $\left(m_{H^{+}}, \tan \beta\right)$ contours by assuming an MSSM model. The result in the $m_{h}-$ max scenario is shown in figure 3 . This figure shows even clearer that the region of low $m_{H^{+}}$and high $\tan \beta$, where most constraints from flavour physics are effective, is indeed the same region where the LHC experiments expect to be sensitive to discovering a charged Higgs boson.

\section{Conclusions}

The charged Higgs boson in the MSSM with MFV has bearing on many low-energy observables currently being probed by experiments. Most notably, input from the $B$-physics program can be used to put strict constraints on $\left(m_{H^{+}}, \tan \beta\right)$. From these constraints, we find that most of the region with low $m_{H^{+}}$and high $\tan \beta$, for which the largest number of $H^{+}$could be produced at 

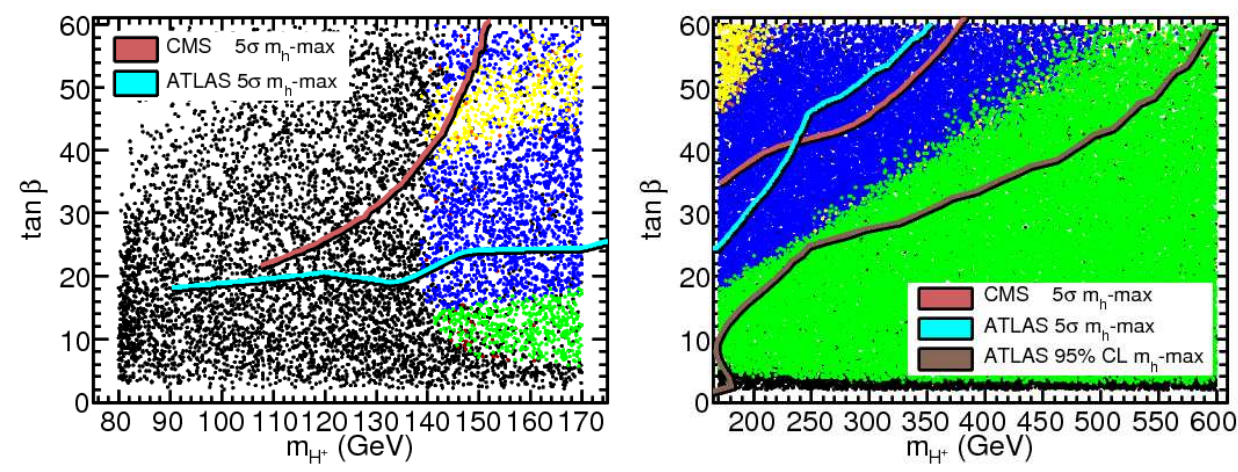

- Allowed

- Direct

- $b \rightarrow s \gamma$

- $\mathrm{B}_{\mathrm{u}} \rightarrow \tau v$

$\mathrm{B}_{\mathrm{s}} \rightarrow \mu^{+} \mu$

- $\mathrm{B} \rightarrow \mathrm{D} \tau \mathrm{v}$

- $\mathrm{K} \rightarrow \mu \mathrm{v}$

Figure 3: Comparison between points from the NUHM scan and the sensitivities of ATLAS and CMS. The experimental contours correspond to the projected sensitivities to discover, or exclude, the MSSM charged Higgs boson in the $m_{h}-$ max scenario. Projection and color coding same as above.

the LHC, is already excluded. This conclusion goes beyond the CMSSM and NUHM scenarios to a large extent, since the most important constraints are obtained from processes with tree-level charged Higgs contributions. It therefore appears unlikely that a light charged Higgs exist with sizable couplings to the fermions. One should of course not trust these indirect experiments blindly, especially since the uncertainties in some cases are still large, but the general indication is clear. As a natural next step, it should be investigated to what extent this picture holds in more general models, including the possibility of non-minimal flavor violation.

\section{Acknowledgments}

I thank my collaborators on this project, David Eriksson and Farvah Nazila Mahmoudi. The valuable input and suggestions provided by the ATLAS charged Higgs group are acknowledged.

\section{References}

[1] D. Eriksson, F. Mahmoudi and O. Stål, Charged Higgs bosons in Minimal Supersymmetry: Updated constraints and experimental prospects, JHEP 11 (2008) 035 [arXiv : 0808 . 3551].

[2] B. C. Allanach, SOFTSUSY: A C++ program for calculating supersymmetric spectra, Comput. Phys. Commun. 143 (2002) 305-331 [hep-ph/ 0104145$].$

[3] F. Mahmoudi, SuperIso v2.3: A program for calculating flavor physics observables in Supersymmetry, arXiv: 0808.3144 .

[4] ATLAS collaboration, Expected Performance of the ATLAS Experiment Detector, Trigger, Physics, CERN-OPEN-2008-020 (2008). To appear.

[5] M. Baarmand, M. Hashemi and A. Nikitenko, Light charged Higgs discovery potential of CMS in the $H^{+} \rightarrow \tau v_{\tau}$ decay with single lepton trigger, J. Phys. G32 (2006) N21. CMS-NOTE-2006-056.

D. Denegri et. al., Summary of the CMS discovery potential for the MSSM SUSY Higgses, hep-ph/0112045.

[6] DØ collaboration, A search for charged Higgs bosons in t $\bar{t}$ events, 2008. DØ Note 5715-CONF. 